\title{
E-MRS Celebrates Silver Anniversary During 2008 Spring Meeting \\ www.emrs-strasbourg.com
}

The 2008 European Materials Research Society (E-MRS) Spring Meeting, held in Strasbourg, France, on May 26-30, marked the 25th anniversary of the founding of the Society in 1983. The Silver Jubilee conference consisted of 17 parallel symposia grouped under five broad headings: Nanomaterials and Nanotechnology, Advanced Functional Materials, Semiconductor Materials, Energy Materials, and Organic/Inorganic Materials and Related Technologies, as well as a plenary session and an industrial exhibition. The conference attracted a record attendance of more than 2,000 participants from approximately 60 countries, with a particularly significant increase in participants from Asia. The conference chairs were Wolfgang Jantsch of the University of Linz, Austria; Francesco Priolo of the University of Catania, Italy; and Hans Richter of Innovations for High Performance Microelectronics, Germany.

\section{Plenary Session}

The plenary session, held on May 28, combined keynote presentations and an awards ceremony. In recognition of the 25th anniversary, Elton N. Kaufmann (Argonne Natl. Lab., USA) opened the session with a brief introduction tracing the development of E-MRS from the seeds sown at the Materials Research Society (MRS) conferences in the early 1980s. He paid tribute to the E-MRS general secretary, Paul Siffert, who was instrumental in advancing the Society's significance in the world's materials scene. E-MRS president Abdelilah Slaoui (CNRS, France), on behalf of the members, presented to Siffert an exquisite marquetry, representing a village near Strasbourg. And a commemorative plaque was presented to E-MRS to mark its anniversary on behalf of the board and members of MRS.

While E-MRS celebrates its silver anniversary, the European Institute of Technology-an initiative of the European Commission-is in the making. Matteo Bonifacio, a policy advisor within the European Commission, detailed the most recent developments. In June, Budapest, Hungary, was announced as the future site of the institute's headquarters, with announcement of the governing board forthcoming.

During the portion of the plenary session on materials science achievements, plenary speaker Sir John B. Pendry, from the Imperial College of London, addressed the topic of metamaterials, a class of materials

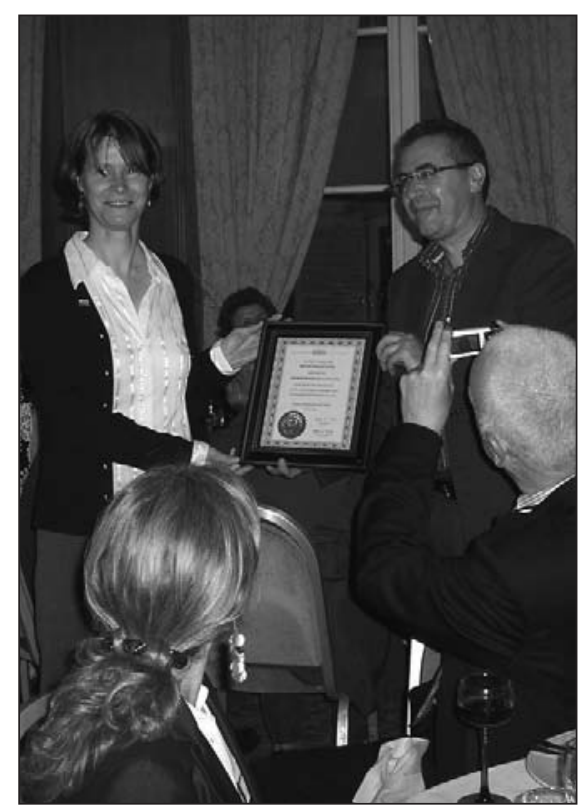

Abdelilah Slaoui (right), the president of the European Materials Research

Society, receives from Cynthia Volkert, president of the Materials Research Society, a special plaque commemorating the 25th anniversary of E-MRS.

that is beyond conventional materials. $\mathrm{He}$ explained that while the properties of conventional materials are derived from their constituent atoms or molecules, in metamaterials, the properties are derived from their constituent units that can be larger than atoms or molecules and that can be engineered. This gives enormous flexibility in designing metamaterials and generates new properties not found in nature.

The best known of these special properties is negative refractive index. Refractive materials provide some limited control of light, but complete control is beyond the scope of simple refracting materials. Manipulation of Maxwell's equation shows that researchers can achieve just that provided they have access to some highly unusual materials properties. The refractive index of a material is a function of its electric and magnetic susceptibilities. To generate a material with negative refractive index, both parameters must be negative.

A potential application for these metamaterials would be to steer light around a hidden region, returning it to its original path on the far side, to which observers would be unaware of the contents of the hidden region, but they would not even be aware that something was hidden: the object would have no shadow. To do that, light must be bent. One of the ways to do that was proposed by Einstein, who Pendry mentioned several times during his lecture; in the general theory of relativity, gravity changes geometry, therefore gravity should bend light. In a similar way, if the coordinates of a material are compressed along one of their axes, the trajectory of a ray that follows the same direction of compression is not affected outside the compressed region, while light is bent in this region and objects inside become invisible, as beams that propagate perpendicular to this direction do not reflect. Pendry concluded his talk by envisioning some of the possibilities that metamaterials may provide in the future, such as the repositioning of objects and the generation of negative space in the laboratory.

Plenary speaker Andre Geim of the University of Manchester discussed his work on the discovery and development of fully two-dimensional (2D) graphene sheets. Graphene sheets are uniquely 2D, unlike thin films that are quasi-2D due to island formations and warping; graphene is wholly flat. Geim produces these 2D materials by flaking off monoatomic layers from a sheet of graphite until all that is left is a single atomic layer of crystalline carbon. This sheet is stable at room temperature, is nearly atomically perfect, and provides a direct visual of the lattice. The ballistic mobility of graphene would suggest it to be a very good material for circuitry. Transistors can be formed in the $10 \mathrm{~nm}$ regime that sustains currents greater than $1 \mu \mathrm{A}$. Of particular interest is the potential to use graphene as an optically transparent conductor similar to indium tin oxide.

The plenary session concluded with award announcements. It is a custom of E-MRS to present a special award every five years. This year, the 25th Anniversary Award was presented to Dan Shechtman of the Technion-Israel Institute of Technology and Iowa State University, by former E-MRS president Hermann Grimmeiss (Lund Univ.). Shechtman received the award for his work on discovering five-fold symmetry crystals (see Sidebar). Student awards from E-MRS and from E-MRS/Elsevier were also presented.

To honor the 25th anniversary of E-MRS, a special celebration was held after the plenary session. The party, "Blue Night," made prominent use of the E-MRS trademark color-blue. Blue beverages, blue 
balloons, blue hats, and a blue crossdressing-mime, were all a part of the party that went on well into the night.

\section{Nanomaterials and Nanotechnology}

In his opening talk in Symposium A on carbon-based nanostructured composite (CBNC) films, H. Hofsäss (Univ. of Göttingen, Germany) guided the audience "beyond the sputtering limit" of advanced deposition techniques: Going beyond the sputtering limit provides a new method for modifying surfaces. Other invited speakers covered the influence of metal concentration on the properties of Me:C films (M. Stüber, Forschungszentrum Karlsruhe, Germany); optical properties of CBNC films (T.P. Nguyen, Inst. des Matériaux Jean Rouxel, Nantes, France); magnetic properties of CBNC films, highlighting possible applications of FePt nanoparticles in the area of high-density magnetic data storage (M. Farle, Univ. Duisburg Essen, Germany); and the electronic structure and electrical properties of
CBNC films, highlighting the importance of morphology in donor-acceptor composite organic solar cells (G. Dennler, Konarka $\mathrm{GmbH}$, Austria).

Other speakers in Symposium A addressed biomedical and biochemical applications. CBNCs are important for biocompatible coatings on medical implants such as artificial joints, or stents in blood vessels. Studies have focused on the tribological behavior of such coatings, and on the ellipsometric characterization of surfaces exposed to a biological environment.

\section{Dan Shechtman Receives E-MRS 25th Anniversary Award}

Dan Shechtman of the Technion-Israel Institute of Technology and Iowa State University received the 25th Anniversary Award from the European Materials Research Society in recognition of his 1982 discovery of the icosahedral phase, the first quasi-periodic crystal.

The origin of the modern science of crystallography can be traced back to 1912 with the seminal x-ray diffraction experiment by von Laue. During the next 70 years, hundreds of thousands of crystals were studied and all were found to be periodic. Over time, since there was no exception, a paradigm developed, that crystals are ordered and periodic. In fact this was the definition of a crystal accepted by the International Union of Crystallographers.

In his talk at the awards ceremony, Shechtman quoted the famous textbook by Kittel "we should not expect the lattice to have a five-fold rotation axis." Indeed, the rotational symmetries allowed in periodic crystals are one, two, three, four, and sixfold. No five-fold and nothing beyond six-fold are allowed. Shechtman's discovery in 1982 of a metallic phase that diffracts electrons, $x$-ray, and neutrons like other crystals but has five-fold rotational axes and overall icosahedral symmetry came therefore as a great surprise to him and to the scientific community.

Soon after the discovery, Shechtman performed a series of experiments in the transmission electron microscope and proved that the newly found crystals are not twins of a periodic phase. Shechtman later collaborated with Ilan Blech of the Technion who proposed a structural model that gave a diffraction pattern that matched the experimental observations, and they both submitted an article to the Journal of Applied Physics in August 1984.

The article was rejected several weeks later as being of no interest to physicists. However, the journal editor suggested submitting the paper to a metallurgical journal. The article was published in Metallurgical Transactions in June 1985, but before that, shortly after the first publication was sent, Shechtman joined forces with John W. Cahn of the National Bureau of Standards and Denis Gratias of the Centre National de la Recherche Scientifique, Vitry, and the four of them sent a condensed article describing the discovery to Physical Review Letters (PRL). This article appeared in PRL in late 1984.

Following the publication of the discovery, a large number of enthusiastic scientists around the world started to study the then available quasi-periodic crystals and to discover new ones. On the other hand, Shechtman's discovery was criticized by many in the scientific community, most and foremost by Linus Pauling, the two-time Nobel laureate. The criticism came mainly from x-ray crystallographers, but in 1987, when single-crystal

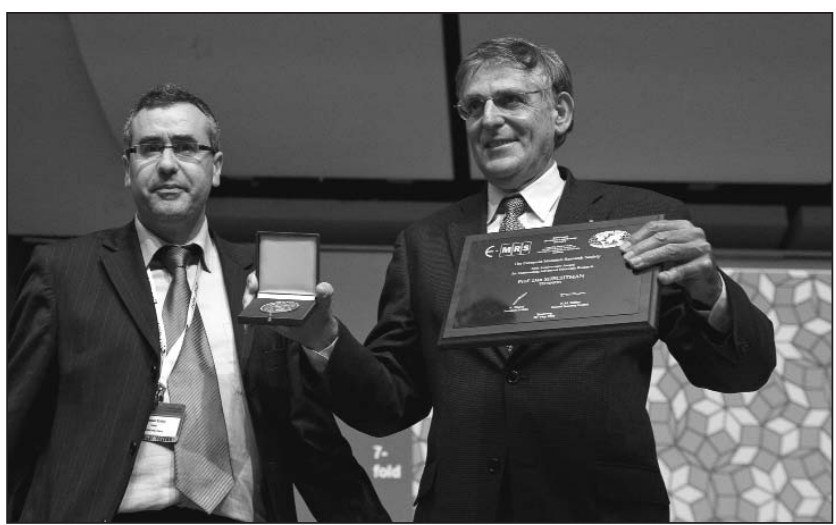

Dan Shechtman (right), of the Technion-Israel Institute of Technology and lowa State University, receives the E-MRS 25th Anniversary Award from E-MRS President Abdelilah Slaoui (CNRS). An image of a quasicrystal is projected in the background.

x-ray patterns of quasi-periodic phases became available, the opposition subsided, and in 1991 the International Union of Crystallographers established a committee to redefine crystal so as to include both periodic and quasi-periodic crystals.

In his talk at the awards ceremony, Shechtman speculated on why it took so long for quasi-periodicity in crystals to be discovered. It is not that five-fold symmetry is rare in crystals; there are hundreds of alloys that have this structure. It is not because they are unstable or that there is only one kind of quasicrystal - there also is decagonal (10-fold) symmetry and several types of icosahedral symmetries; and it is not because these materials are made of exotic elements. Shechtman believes the answer is that the new crystal structure could only be discovered by electron diffraction, since the grains of the material were too small to create a single-crystal diffraction pattern by x-rays.

Furthermore, to most researchers, the types of alloys that have a quasi-periodic structure are generally not very interesting for their limited ductility, and consequently are not commonly investigated. In contradiction to this is the current use of quasicrystals in Maraging stainless steel, which is arguably one of the strongest steels in the world and used in electric razors. Shechtman said that it takes perseverance in pursuing one's observations in the face of derision that makes a discovery possible. 


\section{Closing the Gender Gap in Materials Sciences}

How can women succeed in a male-dominated research environment? What are the hurdles to be taken and what has already been accomplished in the last few decades? These and other questions were the topic of the inaugural Women in Science Apéro-débat "Reaching the Top: Issues and Challenges" held at the 2008 E-MRS Spring Meeting. Co-organized by the European Science Foundation (ESF) and E-MRS, the panel consisted of high-level women in research and science policy who gave not only insight into their often challenging career paths but also valuable advice.

Marja Makarow, the ESF's new and first female Chief Executive, opened the debate. "Diversity in Europe needs to encompass gender diversity," said Makarow. She said that one way toward attaining this diversity is to increase female representation through the introduction of quotas in all public committees, as is the case in Finland (which requires a $40 \%$ quota as a minimum). Previously Vice-Rector of the University of Helsinki and professor of biochemistry and molecular biology, Makarow reported also on the progresses made in Finland, where $28 \%$ of the professors are women. At the ESF, statistics on gender balance within ESF activities such as conferences are being analyzed.

Cynthia Volkert, the current MRS president and a professor of physics at the University of Göttingen, Germany, introduced the mentoring concept to the audience. Her mentoring encompasses, above all, advocating scientific integrity. Recalling her own experiences, Volkert said, "Opportunities are not meted out based only on merit." Subsequently, she recommends finding true mentors where good will can be sensed. As MRS president, she has introduced child care at the conferences, and wants to raise the rather low numbers when it comes to women as symposium organizers, invited speakers, and award recipients. She encourages women to be "part of the solution" by, for instance, nominating other women for awards and leadership positions.

"Women have progressed so much further than I would have ever expected," said Millie Dresselhaus, one of the most prominent members of the materials and physical sciences community. She is the 2007 North American recipient of the L'Oreal UNESCO Award for Women in Science and she is also highly engaged in mentoring. Through informal group mentoring sessions, as well as one-to-one sessions, the first tenured female faculty member in the Massachusetts Institute of Technology (MIT) School of Engineering offers insights on career tips and how to think about science. "There is no one way; I don't give a general formula, but I try to find out what each woman considers as her goal for personal success, what makes her happy," said Dresselhaus. Her own career was far from linear and characterized by changing research fields, which she regards as contributing to her success. In addition to their daily work, Dresselhaus requested that women in leading roles allocate two hours per week to support women in science. Dresselhaus not only works on gaining gender equality, but also extends her mentoring to ethnic minorities.

The panel's final speaker, Caroline Pétigny, is a young project manager at BASF and trained as an organic chemist. Having benefitted from increased equal opportunities in both academia and industry, her way to success was more straightforward, and she elucidated the role of women in the corporate world. Though more women have postgraduate degrees in the life sciences than men, only a minority reach executive positions- $5.6 \%$ at BASF, for example. "Do what you want to do. Be yourself, and be confident in yourself," said Pétigny.

The panelists' overall advice is that women in science should have a certain amount of self-assurance and avoid questioning their abilities to succeed. In response to the call for additional mentoring, E-MRS plans to continue to feature women-in-science panels at future meetings.
A special session entitled "Present and Future of Lasers in Materials Research" was held in Symposium B on May 28 before the plenary session, commemorating the 25th anniversary of E-MRS and its symposia devoted to lasers in materials processing and diagnostics, which were present at the first E-MRS Spring Meeting in 1983. Three invited speakers, J. Schou (Technical Univ. of Denmark), R. Eason (Univ. of Southampton, UK), and M. Farsari (FORTH-Crete, Greece), provided an update on the current status, problems, and perspectives of the basic aspects of lasers in materials research, their capabilities in materials processing, and anticipated future trends. Farsari described different applications of ultrafast lasers for micro- and nanostructuring of materials based on the two-photon polymerization approach. This method constitutes a form of three-dimensional (3D) nonlinear lithography with which it is possible to create structures smaller than those possible by the diffraction limit of light. With this approach, researchers obtained high-quality 3D photonic crystals (PCs) with millimeter scale with submicron resolution, onto which they could also directly write defects and build devices to manipulate light that are not possible to fabricate by other techniques. They also obtained dynamically tunable PCs, and 3D PCs based on nonlinear optical materials. However, the bandgap shift they could achieve with these nonlinear structures was too small, and researchers are still looking for new materials with larger nonlinearity. Farsari also showed how it was possible to functionalize the surface and the bulk of these structures with biomolecules, generating new materials with potential applications in molecular electronics and plasmonics, biosensors, and tissue engineering.

The area of silicon photonics is an expanding research field, which holds the promise of integrating optoelectronic devices on silicon chips-potentially enabling high-performance applications of integrated optics within the mature and inexpensive technology of silicon complementary metal oxide semiconductor (CMOS). Symposium C on Frontiers in Silicon-Based Photonics addressed the fundamental physics and technological aspects of silicon nanomaterials and state-of-the-art applications in silicon lasers, optical sensing, biophotonics, photonic crystal structures, light-emitting devices, and plasmoniccoupled silicon nanodevices.

The opening session, silicon-based nanoplasmonic structures and silicon-based light amplification using nanocrystals and 
rare-earth doping, commenced with the invited talk of A. Polman (AMOLF, The Netherlands). Polman showed that the control of optical fields localized at metaldielectric interfaces (plasmonics), when combined with silicon-based nanostructures, leads to the enhancement of radiative rates in nanocrystals and offers unprecedented possibilities to engineer their emission characteristics. Subsequent talks focused on the ongoing search for optical amplification in silicon nanocrystal waveguides doped with erbium, which show a large potential for the fabrication of compact light amplifiers compatible with silicon technology.

J. Michel (Massachusetts Inst. of Technology, USA) addressed the future challenges of efficient light emission from $\mathrm{Si} / \mathrm{Ge}$ structures and reported preliminary evidences of light amplification in the $1.55 \mu \mathrm{m}$ spectral window. Michel described not only the physics behind advanced germanium devices, but also the engineering considerations for implementing such devices into current CMOS processing schema, and the possible computer architectures such devices could enable. Michel discussed three types of Ge devices: photodetectors, modulators, and lasers. The Ge modulator currently performs at $3 \mathrm{~dB}$ at $1.2 \mathrm{GHz}$; however, as the processing becomes more refined it could operate near $100 \mathrm{GHz}$ while drawing only $50 \mathrm{~W}$. A lasing crystal may be fashioned from Ge by imparting a small amount of tension to alter the bandgap and then doped with $\mathrm{P}$ or As to complete the structure. Michel pointed to the necessity of such devices by considering the future of multicore architecture in computers. He imagined a mesh network of thousands of processors that communicate directly with one another through a photonic mask at speeds near 5000 Tbit/s. The devices he discussed are compatible with current CMOS processing and could be implemented into standard fabrication facilities.

\section{Advanced Functional Materials}

In Symposium E on Thin Films for Large Area Electronics, recent advances in thinfilm transistor (TFT) technologies-particularly in the emerging field of metal-oxide semiconductor TFTs-were reported, including the introduction of ternary alloys as active layers, in which indium was substituted by tin (E. Fortunato, CENIMAT, Portugal), or the use of metal-organic chemical vapor deposition in the fabrication of ZnO TFTs (J. Jo, Ajou University, Korea). U. Chung (Columbia Univ., USA) reported recent developments on linescan sequential lateral solidification (SLS) techniques for polysilicon TFTs, proving the system to be more efficient and more effective for liquid-crystal display manufacturing.

Current limitations of SLS for flat-panel display fabrication and possible solutions also were discussed by H. Kim (Yonsei Univ., Korea). Consistent advancements in the understanding of reliability of $p$-channel polysilicon TFTs were achieved by applying deep level transient spectroscopy (M.A. Exarchos, Univ. of Athens, Greece) and combining bias-temperature-stress and numerical simulations (A. Valletta, IMM-CNR, Italy). Polysilicon TFT technology was demonstrated on polyimide substrates by introducing a combined low-temperature thermal annealing and dehydrogenation/crystallization laser process (L. Mariucci, IMM-CNR, Italy). And organic electronics, as reported by G. Jabbour (Arizona State Univ., USA) is generating increasing attention in view of its continuous improvement in performance coupled with its ability to be deposited on low-cost substrates using solution-based processing.

Symposium G, "Wide Bandgap Semiconductor Nanostructures for Optoelectronic Applications," focused on new III-nitride and $\mathrm{ZnO}$-based nanostructures which already find, or may find in the near future, applications in optoelectronic devices. A majority of presentations covered the self-organized growth and the characterization of nanorods that can be produced with high structural perfection. Strong photoluminescence and electroluminescence from assemblies of these nanostructures were reported and a demonstration of LED devices was revealed.

A key issue with III-nitride quantum structures is to avoid internal polarization fields; either by growth on hexagonal aor m-plane substrates or using the metastable, cubic configuration of the nitrides. Both strategies, together with new microcavity-based nanostructures, were addressed in a number of presentations during the symposium. Another highlight of the symposium was the observation of the strong coupling regime in a III-nitride microcavity at room temperature and the demonstration of BoseEinstein condensation with this device. Impressive progress also was made with III-nitride inter-subband devices for $1.55 \mu \mathrm{m}$ detection. These detectors reach cut-off-frequencies in the $100 \mathrm{GHz}$ region and thus may allow extending the bit rate in information networks.

\section{Semiconductor Materials}

M. Hytch created a buzz amongst the Symposium I audience members when he detailed groundbreaking work that he and his co-workers are doing at CEMES-CNRS in Toulouse. Hytch and his co-workers have found a method for quantitative strain mapping at the nanoscale in electronic devices using electron microscopy. Also, C. Claeys from IMEC showed how the integration of Ge into Si technology can successfully meet the International Technology Roadmap for Semiconductors' requirement for future device generations, and identified the chances and challenges.

Symposium J, entitled "Beyond Silicon Technology: Materials and Devices for Post-Si CMOS," was intended to be a vehicle for exploring how the materials and related logic systems might evolve in the mid to long term. It was planned in conjunction with Symposium I, which looked at shorter term issues, and Symposium K, which focused on silicon materials. The first session covered alternative logic, long-term approaches, and blue sky projects. Among the issues considered in the session were cellular automata, carbon nanotubes, polymers, and graphene. However, there was a near consensus in the symposium that targets of $15 \mathrm{~nm}$ and beyond are unlikely to be met by silicon and, in the medium term, the most likely option is to replace the silicon channel in $p$ MOS with germanium. The direction for $n \mathrm{MOS}$ is more debatable, though III-Vs, germanium, and SiGe are options.

Also covered in Symposium J, J.R. Barker (Univ. of Glasgow, UK) talked about his creation of a set of algorithms (written in Fortran) that enable a bank of up to 100 computer processors (not supercomputers) to calculate the charge distribution and current flow in ultrasmall devices in approximately one hour. Barker is aiming toward being able to determine these operating parameters for the $4 \mathrm{~nm}$ gate length devices that already exist in the laboratory-which manufacturers will be fabricating by 2020. At present, Barker believes that full atomistic models are nearly achievable. The calculating engine, Barker said, is a "self-consistent 3D device simulator based on parallelized, recursive nonequilibrium green function quantum transport methodology to study this problem nonperturbatively." Barker is able to determine the current flow in a device containing a single dopant atom and showed images of the electron flow around the atom. "A surprising result is a reduction in variability predicted for very short channel devices," he said. These calculations are powerful and provide a valuable tool toward the understanding of nanoscale devices.

Symposium K, “Advanced Silicon 
Materials Research for Electronic and Photovoltaic Applications," brought together scientists from industry and basic research, creating a forum to discuss the results of recent research along with potential applications for electronic and solar silicon.

Following the joint session with symposia I and J, M. Kondo from the National Institute of Advanced Industrial Science and Technology, Japan, informed the audience that nanocrystalline silicon is a promising material for low-cost, high-efficiency solar cells.

H.J. Queisser (Max Plank Inst, for Solid State Research, Germany), a pioneer in the field, presented an overview on overcoming limits for solar cell efficiency. As solar cells have now become an economical and political issue, their improvement is important. Though lifetime is no longer a limitation, Queisser stressed how critical it was to determine the lifetime of electrons in the early days of semiconductor technology. However, the principle of detailed balance to derive the thermodynamic limit for the energy conversion efficiency of a semiconductor junction photovoltaic, which Queisser and Shockley used for the first time in 1961, is still valid today. By reconsidering the assumptions made in this early work, Queisser exposed the modern efforts of cell development used to overcome this detailed balance limit. Queisser and Shockley started by considering having only one Sun; however, by using concentrated illumination, higher energy output could be delivered. They also considered having only one $p-n$ junction. Presently, multijunction cells can be fabricated delivering high energy output, although they are expensive. The third assumption made was considering only one pair per photon. Attempts have since been made to more fully utilize the energies of the photons in the blue spectral regime of sunlight. The last assumption by Queisser and Shockley was to not consider the existence of deep centers. However, these centers would allow absorption of red photons, further increasing their efficiency.

\section{Energy Materials}

Competitive with current Si-waferbased modules, a $30 \times 30 \mathrm{~cm}^{2} \mathrm{Cu}(\mathrm{In}, \mathrm{Ga})$ $(\mathrm{Se}, \mathrm{S})_{2}$ CIGS sub-module with $15 \%$ efficiency was reported in Symposium L, "Thin Film Chalcogenide Photovoltaic Materials." CdTe commercialization has achieved costs of $1.09 \$ / W_{p}$, the lowest figure in the market. During discussions concerning the "road map to higher efficiencies," optical losses were considered responsible for the gap between cell and

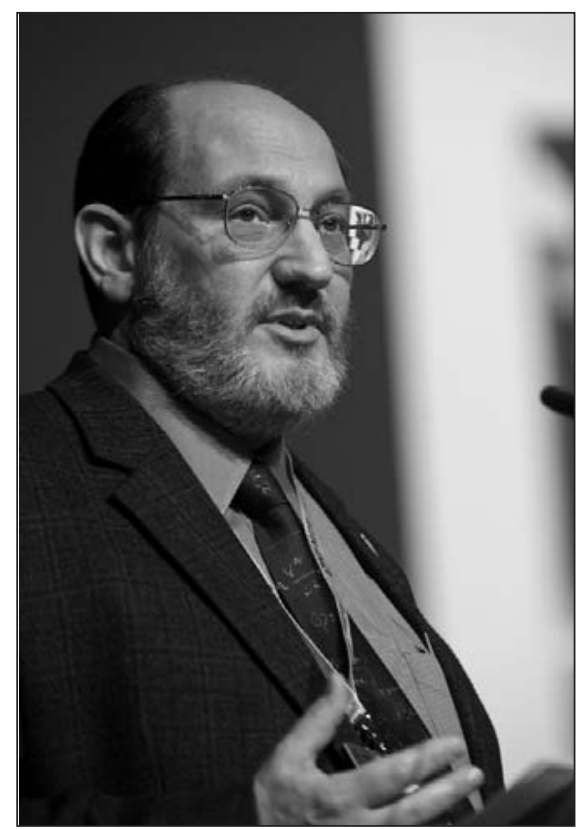

Elton N. Kaufmann (Argonne National Laboratory) traces the development of E-MRS.

module efficiencies. The recently published $19.9 \%$ efficiency of CIGS cells will serve as a benchmark for the chalcogenide community.

Thin film growth processes were among the important topics discussed during Symposium L. Stabilizing elemental $\mathrm{Cu}, \mathrm{In}, \mathrm{Ga}, \mathrm{Se}, \mathrm{S}$ in a solvent and subsequent annealing were presented as a novel method, solving the problem of a nonhomogeneous Ga profile often encountered during sequential $\mathrm{Cu}(\mathrm{In}, \mathrm{Ga})(\mathrm{Se}, \mathrm{S})_{2}$ formation. However, a refined temperature profile during metallic precursor chalcogenization also appears important. For CdTe growth, a low temperature process window for oriented crystallization was presented, which may lead to denser films and could allow the CdTe thickness in the device to be reduced.

The large amount of energy available from waste-heat sources has increased the scientific community's interest in thermoelectrical methods to convert this energy into electricity. However, the performance of materials and their environmental impact appear to be the primary limitations in the use of thermoelectric generators. Symposium M, "Unconventional Thermoelectrics: From New Materials to Energy Conversion Devices," was intended to bring together scientists involved in the search for new bulk or nanostructured thermoelectric materials to address the aforementioned limitations.
Symposium N, "Nuclear Materials," covered issues including materials for advanced fusion systems; materials for spallation and transmutation; materials for GEN IV reactors; structural materials for GEN II and III reactors; fuel materials, fuel matrix and target for transmutation in reactors; and waste form materials for geological disposal or to be reprocessed. The session on fusion addressed RAFM/ RAF steels, their ODS versions, $\mathrm{SiC} / \mathrm{SiC}$, $\mathrm{SiC} / \mathrm{C}$, and $\mathrm{C}$ versions for light elements on the armor wall, as well as tungsten on the diverter. Helium behavior in the system remains a crucial aspect.

\section{Organic-Inorganic Materials and Related Technologies}

The field of organic and hybrid materials is expanding rapidly with the use of nano-objects considered as building blocks in the elaboration of functional materials. The design of these nanoobjects with adequately surface properties is of primary importance in the development of new nano-devices. In Symposium P, "Advanced Organic and/or Inorganic Functional Materials," M.P. Pileni (Paris) demonstrated that the physical properties of isolated nanocrystals and self-organized 2D and 3D superlattices are markedly different. She described a "new" physics with collective optical and magnetic properties, due to the nanocrystal organization. G. Decher (Strasbourg) presented a multi-step assembly method, the so-called layer-bylayer (LbL) deposition technique, which is an easy process for the fabrication of multi-composite films. Furthermore, L. Rozes (Paris) demonstrated that the control of the hybrid organic-inorganic interface is a key parameter to tune the properties of hybrid nanocomposites by detailing different examples of hybrid materials based on nanoparticles or metallic oxo-clusters.

Processing methods toward nanodevices was another significant topic covered during Symposium P. D. Goldberg (Natl. Inst. for Materials Science, Tsukuba, Japan) presented novel nanotubes (NTs) produced at the Nanoscale Materials Center, principally concerning BN NTs, using high-temperature synthetic methods, primarily induction heating. These NTs show higher chemical and oxidation stability than carbon NTs, and are suitable for high temperature applications. The BN NTs can adsorb hydrogen up to 4.2 wt \%, and their electrical properties can be tuned by doping. They can be heavily deformed, yet recover the initial shape after the strength is released. Goldberg showed how polystyrene composite films were 
generated with these BN NTs with enhanced elasticity and improved thermal stability, ceramic materials with improved plasticity, and DNA ensembles that can be selectively deposited onto gold.

M. Barboiu, from the Institut Europeen des Membranes, Montpellier, France, focused on functional membranes in which the recognition-driven transport properties could be ensured by a well-defined incorporation of receptors of specific molecular recognition and self-organization functions, incorporated in a hybrid dense or mesoporous materials. The best example of such membranes is the cell membrane. With these strategies, researchers are approaching functional constitutional devices in which the self-assembly with selection or dynamic combinatorial chemistry plays a leading role. The researchers' philosophy is to choose the initial building blocks to generate a library of interexchanging species, which will be selected under constitutional internal or external pressures, favoring some suprastructures in front of the others. To illustrate this concept, Barboiu presented results obtained by combining adenine and uracil, opening new avenues toward a constitutional transcription of basepairing codes in hybrid materials.

In Symposium Q, "Functional Supramolecular Architectures for Organic Electronics and Nanotechnology," Nobel laureate J.-M. Lehn discussed the design, selection, and adaptation of functional nanostructures through self-organization mechanisms. Supramolecular chemistry uses molecular recognition mechanisms directed by self-organization to create functional supramolecular architectures. A key point in this approach is using metal cations, called "ion dots," as addressable centers for nanoscience. This allows the formation of grids of metals combined with different conjugated ligand organic molecules containing $\mathrm{N}$ atoms that show significant supramolecular electronics and spintronics properties.

Lehn plays with chemistry by introducing different species in selected sites through toposelective synthesis processes.
This tool allows for toposelective modulation of optical, electronic, and magnetic properties by external triggers/effectors, such as the $\mathrm{pH}$ of the solution or the protonic modulation of redox properties. Lehn understands nanoscience and nanotechnology as programmed self-organization, and chemistry is the science to show it through processes such as the spontaneous but controlled generation of well-defined, large, complex, organized, functional supramolecular architectures.

For further details on the research results reported at the 2008 E-MRS Spring Meeting, see the E-MRS Web site at www.emrs-strasbourg.com as well as additional meeting highlights provided by the MRS Meeting Scenes, posted at Web site www.mrs.org. The next E-MRS Spring Meeting will be held in Strasbourg, France, on June 8-12, 2009.

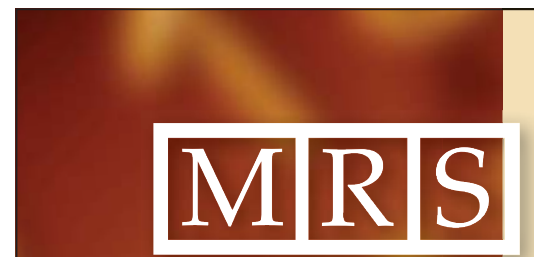

\section{Upcoming Meetings and Workshops of Interest to the Materials Research Community}

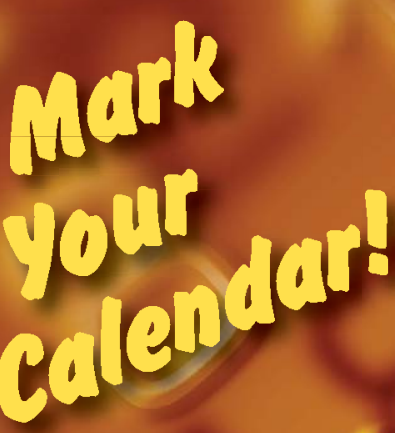

April 13-17, 2009 2009 MRS Spring Meeting \& Exhibit

Moscone West and San Francisco Marriott Hotel • San Francisco, California, USA

May 3-7, 2009 - International Conference on Neutron Scattering (ICNS 2009)

Hosted by the Neutron Scattering Society of America

Knoxville Convention Center $\cdot$ Knoxville, Tennessee, USA

June 7-11, 2009 - New Diamond and Nano Carbons Conference (NDNC 2009)

Grand Traverse Resort \& Spa • Traverse City, Michigan, USA

July 6-9, 2009 - Organic Microelectronics \& Optoelectronics V

A joint Workshop of ACS - IEEE - MRS

San Francisco Marriott Hotel • San Francisco, California, USA

August 16-20, 2009 XVIII International Materials Research Congress 2009

Organized by the Sociedad Mexicana de Materiales (MRS-Mexico);

co-sponsored by the Materials Research Society $\cdot$ Cancun, Mexico

November 30-December 4, 2009 - 2009 MRS Fall Meeting \& Exhibit

Hynes Convention Center and Sheraton Boston Hotel • Boston, Massachusetts, USA

For more information visit www.mrs.org/meetings 